\title{
Capacity Simulation and Analysis of an IEEE 802.11n System in a Residential House
}

\author{
E. Tanghe ${ }^{1}$, D. P. Gaillot ${ }^{2}$, W. Joseph ${ }^{1}$, M. Liénard ${ }^{2}$, W. De Ketelaere ${ }^{3}$, L. Martens ${ }^{1}$ \\ ${ }_{1}$ iMinds-INTEC/WiCa, Ghent University, Ghent, Belgium, emmeric.tanghe@intec.ugent.be \\ ${ }^{2}$ IEMN/TELICE, University of Lille 1, Lille, France, davy.gaillot@univ-lille1.fr \\ ${ }^{3}$ Excentis, Ghent, Belgium, wim.deketelaere@excentis.com
}

\begin{abstract}
This paper presents an analysis of the MultipleInput Multiple-Output (MIMO) capacity based on channel sounding measurements in a residential house. Specular and dense multipath components are estimated from the channel sounding data with the RiMAX maximum-likelihood algorithm. These multipath channels serve as input for the capacity calculations. Specifically, capacity calculations are done for a $2 \times 2$ MIMO system that uses the legacy mode of the IEEE 802.11n standard.
\end{abstract}

Index Terms-channel sounding, multipath estimation, specular multipath, dense multipath, residential environment, capacity, IEEE 802.11n

\section{INTRODUCTION}

The objective of this paper is to create a framework for the simulation of IEEE $802.11 \mathrm{n}$ capacity between different locations in a residential house. The IEEE 802.11n system operates in the $2.4 \mathrm{GHz}$ frequency band. The capacity is calculated using measurement-based multipath radio channels. The framework allows us to adjust the physical-layer parameters of IEEE 802.11n (FFT size, guard interval, etc.). The geometrical configuration of the antenna arrays at access point and client side also need to be specified, together with the polarimetric complex antenna radiation patterns.

The multipath radio channels in this work are based on radio channel sounding experiments with a vector network analyzer and virtual antenna arrays. Multipath components are estimated from the sounding data with the RiMAX maximumlikelihood algorithm [1]. The multipath radio channel contains both Specular Multipath Components (SMCs) and Dense Multipath Components (DMC). The inclusion of DMC in radio channel models has been pushed forward by the European COST actions 2100 and IC1004. It stems from the observation that SMCs alone are often insufficient to account for all multipath energy present in a radio channel. Capacity analyses of DMC-inclusive radio channels are scarce in literature: examples can be found in [2] and [3].

\section{MEASUREMENT CAMPAigN}

Frequency-domain channel sounding measurements were performed in a fully detached residential house. The house is located in a rural area devoid of any close-by wireless networks that may cause interference with the measurements. A Vector Network Analyzer (VNA) of type Rohde \& Schwarz ZNB20 was used to probe the radio channel in a $80 \mathrm{MHz}$ bandwidth centered around $2.51 \mathrm{GHz}$. In this frequency band, $M_{F}=200$ uniformly spaced frequency points were sampled. The feeder cables for the transmitting antenna (Tx) and the receiving antenna $(\mathrm{Rx})$ were included in the VNA calibration so they do not appear in the measurement data.

At both link ends, a virtual antenna array was created by an automated positioning system. At each link end, the virtual array consists of two planar horizontal Uniform Circular Arrays (UCAs) of eight antennas each. Both UCAs are stacked vertically. Thus, at the transmitting (receiving) side, the complete virtual array consists of $M_{T}=16\left(M_{R}=16\right)$ antenna elements. For each position of transmit and receive antenna, the VNA took one sweep of the frequency range. As transmitting and receiving antenna, dual-polarized patch antennas were used to access the horizontal $(\mathrm{H})$ and vertical (V) component of the electric field at transmitter and receiver separately. Programmable electronic switches were used to switch between the $\mathrm{H}$ and $\mathrm{V}$ ports of the measurement antennas during measurements. At each link end, the lowest position of the patch antennas was $0.80 \mathrm{~m}$ above ground level. Measurements were done without any people present in the house because frequency-swept measurements with virtual antenna arrays require a static radio channel without movement.

The residential house is made up of two equally sized floors. Fig. 1 presents the floor plan of the ground floor. In total, 16 Tx-Rx links were measured around the house. For the first eight links, both the Tx and Rx were located on the ground floor. The Tx remained fixed in the lower-left corner in Fig. 1, while the Rx was moved to eight locations distributed across 
the different rooms (both line-of-sight and non-line-of-sight situations). For the last eight links, the $\mathrm{Tx}$ was moved to a fixed location on the first floor, while the $\mathrm{Rx}$ was moved to the same eight locations on the ground floor as for the first eight links (strictly non-line-of-sight situations).

\section{DATA MODEL AND MULTIPATH ESTIMATOR}

\section{A. Data model}

The measured polarimetric array response vectors $h_{X Y} \in$ $\mathbb{C}^{M_{R} M_{T} M_{f} \times 1}$ can be written as the sum of an SMC part $s_{X Y}$, a DMC part $\boldsymbol{d}_{X Y}$, and a measurement noise part $\boldsymbol{n}_{X Y}$ :

$$
\boldsymbol{h}_{X Y}=\boldsymbol{s}_{X Y}\left(\boldsymbol{\theta}_{s, X Y}\right)+\boldsymbol{d}_{X Y}\left(\boldsymbol{\theta}_{d, X Y}\right)+\boldsymbol{n}_{X Y}\left(\sigma_{X Y}^{2}\right)
$$

In this work, the subscripts $X$ and $Y$ denote the polarization of the transmitting and the receiving antenna, respectively. $X$ and $Y$ are either horizontal $(H)$ or vertical $(V)$. It is generally assumed that $\boldsymbol{h}_{X Y}$ follows a multivariate circularly symmetric complex Gaussian distribution [1]:

$$
\boldsymbol{h}_{X Y} \sim \mathcal{N}_{c}\left(\boldsymbol{s}_{X Y}\left(\boldsymbol{\theta}_{s, X Y}\right), \boldsymbol{R}_{X Y}\left(\boldsymbol{\theta}_{d, X Y}, \sigma_{X Y}^{2}\right)\right) .
$$

The parameter vector $\boldsymbol{\theta}_{s, X Y}$ groups the parameters associated with the SMCs $s_{X Y}$. On one hand, these include the geometrical parameters: the angles of arrival/departure and the times of arrival. The geometrical SMC parameters are identical across all four polarization subchannels $X Y$. On the other hand, $\boldsymbol{\theta}_{s, X Y}$ also includes the complex amplitudes $\gamma_{X Y}$ of the SMCs. The complex amplitudes differ between different polarization subchannels because of the polarizationdependency of electromagnetic interactions.

The DMC $\boldsymbol{d}_{X Y}$ and the noise $\boldsymbol{n}_{X Y}$ in (1) are fully determined by the covariance matrix $\boldsymbol{R}_{X Y}$ in (2). This covariance matrix is assumed to have the following structure involving Kronecker products [1]:

$$
\begin{aligned}
& \boldsymbol{R}_{X Y}\left(\boldsymbol{\theta}_{d, X Y}, \sigma_{X Y}^{2}\right) \\
& =\boldsymbol{R}_{d, X Y}\left(\boldsymbol{\theta}_{d, X Y}\right)+\sigma_{X Y}^{2} \boldsymbol{I}_{M} \\
& =\boldsymbol{I}_{M_{R}} \otimes \boldsymbol{I}_{M_{T}} \otimes \boldsymbol{R}_{f, X Y}\left(\boldsymbol{\theta}_{d, X Y}\right)+\sigma_{X Y}^{2} \boldsymbol{I}_{M} .
\end{aligned}
$$

In (3), $\boldsymbol{I}_{\kappa}$ is the identity matrix of size $\kappa$ and $M=$ $M_{R} M_{T} M_{f}$. From (3), it is clear that the measurement noise is modeled as independent and identically distributed (i.i.d.) complex Gaussian noise with variance $\sigma_{X Y}^{2}$. Furthermore in (3), the DMC covariance matrix $\boldsymbol{R}_{d, X Y}$ is modeled as uncorrelated in the spatial domains at the receiving side $\left(\boldsymbol{I}_{M_{R}}\right)$ and at the transmitting side $\left(\boldsymbol{I}_{M_{T}}\right)$, and as correlated in the frequency domain $\left(\boldsymbol{R}_{f, X Y}\right)$. The exact mathematical structure of $\boldsymbol{R}_{f, X Y}$ is derived from the DMC power delay profile
$\psi_{X Y}(\tau)$ as function of delay $\tau$. It is assumed that the DMC power delay profile follows the well-known exponential decay:

$$
\psi_{X Y}(\tau)=\alpha_{d, X Y} e^{-\beta_{d, X Y}\left(\tau-\tau_{d, X Y}\right)} .
$$

In (4), $\alpha_{d, X Y}, \beta_{d, X Y}$, and $\tau_{d, X Y}$ are three parameters which fully describe the DMC of polarization subchannel $X Y$ and are gathered into the DMC parameter vector $\boldsymbol{\theta}_{d, X Y}$.

\section{B. RiMAX estimator}

The RiMAX estimation algorithm is applied to the channel sounding data to obtain maximum-likelihood estimates $\hat{\boldsymbol{\theta}}_{s, X Y}$ and $\hat{\boldsymbol{\theta}}_{d, X Y}$ of the SMC and DMC multipath parameters, and $\hat{\sigma}_{X Y}^{2}$ of the noise variance [1]. The RiMAX algorithm was extended with the Effective Aperture Distribution Function (EADF) framework [4]. The EADF allows us, during estimation, to account for and exclude the effect of the dual-polarized complex radiation patterns of the measurement antennas.

The RiMAX algorithm is an iterative algorithm: in each iteration, a set of five SMCs is initialized using a multidimensional beamformer and optimized using the fast-converging Levenberg-Marquardt algorithm. Subsequently, the DMC and noise, i.e., the measured channel minus all optimized SMCs, are fitted to the data model in (3) and (4). Furthermore, the reliability of each estimated SMC is checked against a criterion based on the SMC power estimate and its estimation error variance (see [1] and [5] for details). If all five SMCs in one RiMAX iteration fail the reliability criterion, then the algorithm is stopped.

Fig. 1 shows the estimated SMCs of one of the links where both Tx and Rx are on the ground floor and in non-line-of-sight of each other. SMCs emanating from the Tx and impinging on the Rx are displayed in blue and red, respectively. The length of the SMC lines corresponds to the path length of each individual multipath component. The thickness of the SMC lines is proportional to the power of the paths. For the Tx-Rx link in Fig. 1, the RiMAX algorithm stopped after it found $P=98$ SMCs. For all 16 Tx-Rx links, the number of SMCs $P$ varied between 11 and 155 .

\section{CAPACITY SIMULATION}

\section{A. IEEE 802.11n legacy mode system}

The capacity simulation is done for a communication system that adheres to the IEEE 802.11n wireless standard. Channel 6 in the $2.4 \mathrm{GHz}$ ISM band is chosen for the communication link (center frequency is $2.437 \mathrm{GHz}$ and bandwidth is $20 \mathrm{MHz}$ ). The system under consideration employs two antennas at both the receiving and the transmitting side $\left(M_{R}^{\prime}=M_{T}^{\prime}=2\right)$. At both link ends, the two-antenna arrays are centered at exactly the same Tx-Rx locations used in the channel sounding 


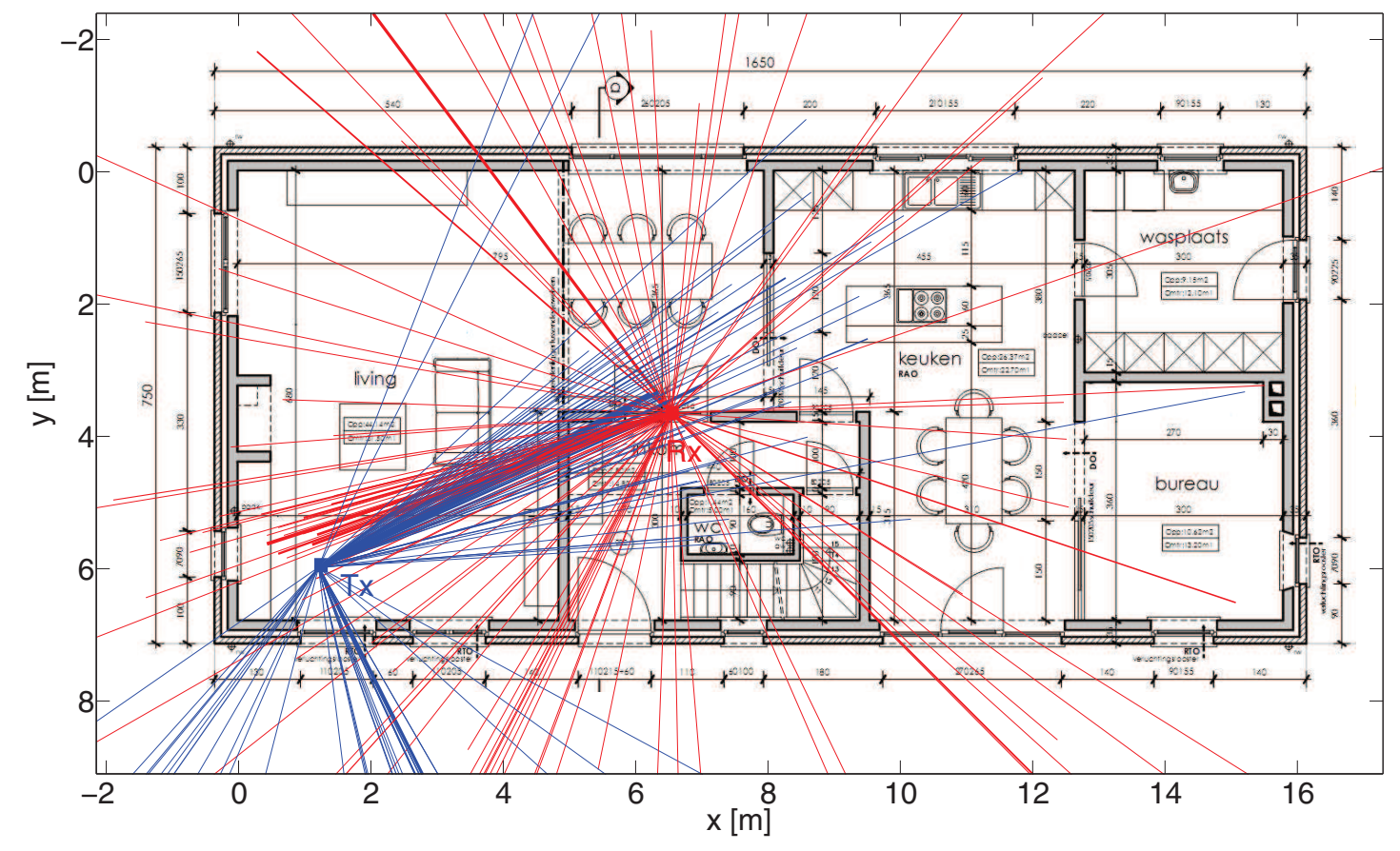

Fig. 1. Floor plan + estimated SMCs of one of the Tx-Rx links

campaign. The two antennas are separated along the $\mathrm{x}$-axis in Fig. 1 by half a wavelength corresponding to the channel 6 center frequency. All four antennas are vertically polarized half-wave dipole antennas with a gain of $2.15 \mathrm{dBi}$.

In legacy mode, IEEE 802.11n OFDM uses a 64-point FFT in a $20 \mathrm{MHz}$ bandwidth. Of these 64 OFDM subcarriers, 52 are datacarriers. For the capacity simulation, the radio channel is simulated only in the $M_{f}^{\prime}=52$ datacarriers. Finally, the capacity calculation also accounts for the $800 \mathrm{~ns}$ guard interval used in IEEE 802.11n legacy mode. This guard interval increases the OFDM symbol duration $T_{s}$ to $4 \mu \mathrm{s}$, which corresponds to a symbol rate $f_{s}$ of $250 \mathrm{ksymbols} / \mathrm{s}$.

\section{B. Radio channel simulation}

Radio channel response vectors ${ }^{1} \boldsymbol{h}^{\prime} \in \mathbb{C}^{M_{R}^{\prime} M_{T}^{\prime} M_{f}^{\prime} \times 1}$ are generated based on the specifications in Section IV-A. The response vectors are written as the sum of an SMC part $s^{\prime}$ and a DMC part $\boldsymbol{d}^{\prime}$, i.e.,

$$
h^{\prime}=s^{\prime}+d^{\prime}
$$

The SMC part $\boldsymbol{s}^{\prime}$ is calculated as:

\footnotetext{
${ }^{1}$ In this section, the prime symbol is used to denote variables that follow from radio channel simulation. This is to distinguish them from the same variables without prime that are associated with the channel sounding and estimation procedures in Sections II and III.
}

$$
\boldsymbol{s}^{\prime}=\sum_{\substack{X \in\{H, V\} \\ Y \in\{H, V\}}}\left[\left(\boldsymbol{G}_{R, Y}^{\prime} \circ \boldsymbol{A}_{R}^{\prime}\right) *\left(\boldsymbol{G}_{T, X}^{\prime} \circ \boldsymbol{A}_{T}^{\prime}\right) * \boldsymbol{A}_{f}^{\prime}\right] \hat{\gamma}_{X Y}
$$

In (6), the $\circ$ and $*$ operators denote the Schur-Hadamard and the column-wise Khatri-Rao matrix products, respectively. $\boldsymbol{A}_{R}^{\prime} \in \mathbb{C}^{M_{R}^{\prime} \times P}, \boldsymbol{A}_{T}^{\prime} \in \mathbb{C}^{M_{T}^{\prime} \times P}$, and $\boldsymbol{A}_{f}^{\prime} \in \mathbb{C}^{M_{f}^{\prime} \times P}$ are steering matrices containing complex exponentials. $\boldsymbol{A}_{R}^{\prime}$ depends on the Rx array's geometry and on the SMC angles of arrival. Similarly, $\boldsymbol{A}_{T}^{\prime}$ is dictated by the Tx array's geometry and SMC angles of departure. The steering matrix $\boldsymbol{A}_{f}^{\prime}$ depends on the simulated frequency points and the SMC times of arrival. Furthermore, $\boldsymbol{G}_{R, Y}^{\prime}$ and $\boldsymbol{G}_{T, X}^{\prime}$ are matrices that premultiply the complex exponentials in the $\mathrm{Rx}$ and $\mathrm{Tx}$ steering matrices with the appropriate polarimetric antenna gains. Lastly, $\hat{\gamma}_{X Y} \in \mathbb{C}^{P \times 1}$ contains the SMC estimated complex amplitudes in polarization subchannel $X Y$. The sum in (6) is taken over all four polarization subchannels.

To simulate the DMC part $\boldsymbol{d}^{\prime}$ in (5), we first reconstruct DMC covariance matrix $\boldsymbol{R}_{d, X Y}^{\prime} \in \mathbb{C}^{M^{\prime} \times M^{\prime}}$ based on the structure in (3):

$$
\boldsymbol{R}_{d, X Y}^{\prime}=\boldsymbol{I}_{M_{R}^{\prime}} \otimes \boldsymbol{I}_{M_{T}^{\prime}} \otimes \boldsymbol{R}_{f, X Y}^{\prime}\left(\hat{\boldsymbol{\theta}}_{d, X Y}\right)
$$

Following, the simulated DMC covariance matrix is subjected to an eigendecomposition: 


$$
\boldsymbol{R}_{d, X Y}^{\prime}=\boldsymbol{U}_{X Y} \boldsymbol{\Lambda}_{X Y} \boldsymbol{U}_{X Y}^{\dagger}
$$

In (8), $\dagger$ denotes the Hermitian transpose. The columns of $\boldsymbol{U}_{X Y}$ contain the eigenvectors of $\boldsymbol{R}_{d, X Y}^{\prime}$, while $\boldsymbol{\Lambda}_{X Y}$ is a diagonal matrix of real non-negative eigenvalues. The eigendecomposition is then used to generate observations of $\boldsymbol{d}^{\prime}$ :

$$
\boldsymbol{d}^{\prime}=\sum_{\substack{X \in\{H, V\} \\ Y \in\{H, V\}}}\left(\boldsymbol{U}_{X Y} \boldsymbol{\Lambda}_{X Y}^{1 / 2}\right) \boldsymbol{z}_{X Y}
$$

In (9), $\boldsymbol{z}_{X Y} \in \mathbb{C}^{M_{R}^{\prime} M_{T}^{\prime} M_{f}^{\prime} \times 1}$ is a vector of i.i.d. complex Gaussian random variables with unit variance. For each Tx-Rx link, 200 independent samples of $\boldsymbol{h}^{\prime}$ are simulated. It is noted that, for the specific simulation configuration with vertically polarized dipole antennas described in Section IV-A, the sums in (6) and (9) only contain one of the four usual terms, i.e., the term for which $X=Y=V$.

\section{Capacity calculation}

For the calculation of capacity, the simulated channel response vector $\boldsymbol{h}^{\prime}$ is first converted into $M_{f}^{\prime}$ channel matrices $\boldsymbol{H}_{i}^{\prime} \in \mathbb{C}^{M_{R}^{\prime} \times M_{T}^{\prime}}$, where $i$ denotes the OFDM subcarrier. The capacity $C^{\prime}$ of the simulated channel is then computed by summing the partial capacities of each individual OFDM subcarrier:

$$
C^{\prime}=\sum_{i=1}^{M_{f}^{\prime}} \mathbb{E}\left[f_{s} \log _{2}\left(\operatorname{det}\left(\boldsymbol{I}_{M_{R}^{\prime}}+\frac{\rho}{M_{T}^{\prime}} \boldsymbol{H}_{i}^{\prime}\left(\boldsymbol{H}_{i}^{\prime}\right)^{\dagger}\right)\right)\right]
$$

In (10), $\rho$ is the Signal-to-Noise Ratio (SNR) at one of the receiving antennas, averaged over all OFDM subcarriers. The expectation $\mathbb{E}[\cdot]$ is taken over the 200 independent realizations of the simulated channel. Commonly, the channel matrices $\boldsymbol{H}_{i}^{\prime}$ are normalized. We chose the normalization such that the expected value of each element of $\boldsymbol{h}^{\prime}$ equals one, or equivalently,

$$
\sum_{m=1}^{M_{R}^{\prime}} \sum_{n=1}^{M_{T}^{\prime}} \mathbb{E}\left[\left|\boldsymbol{H}_{i}^{\prime}(m, n)\right|^{2}\right]=\frac{M_{R}^{\prime} M_{T}^{\prime}}{M_{f}^{\prime}}
$$

Fig. 2 shows the simulated IEEE $802.11 \mathrm{n}$ capacity $C^{\prime}$ versus SNR $\rho$ of the same Tx-Rx link as in Fig. 1 (black curve). For comparison, the capacity curves of IEEE $802.11 \mathrm{n} 2 \times 2$ MIMO systems with fully independent and fully correlated spatial streams are also shown (blue and red curves). The magenta curve shows the capacity of an IEEE 802.11n Single-Input Single-Output (SISO) system with one spatial stream.

It is clear from Fig. 2 that the simulated capacity of the Tx-Rx link under consideration is close to the capacity of two

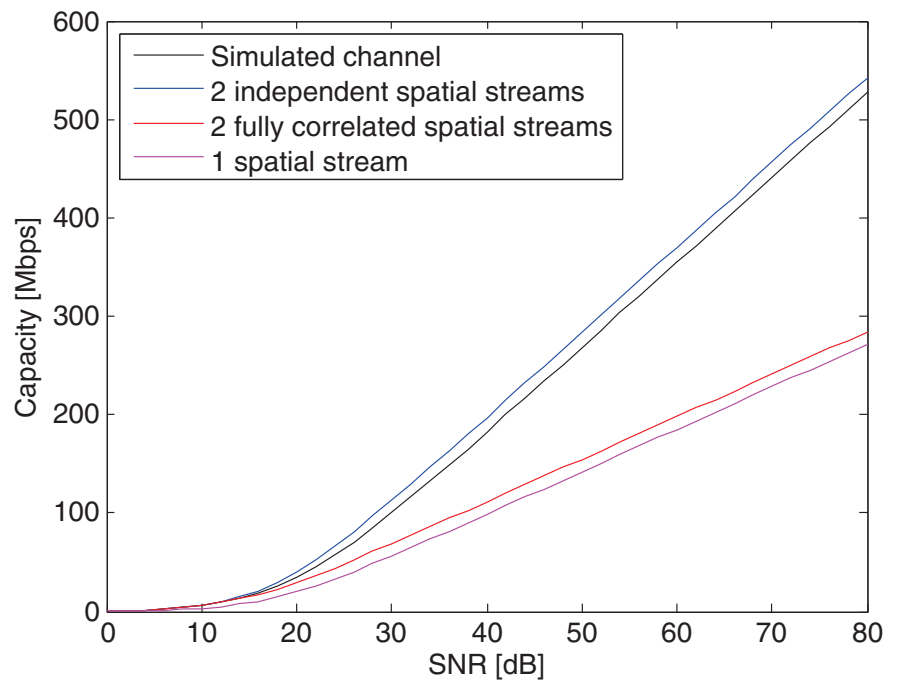

Fig. 2. Capacity versus SNR for the Tx-Rx link in Fig. 1

independent spatial streams. This is to be expected by considering Fig. 1. Fig. 1 shows a multipath-rich non-line-of-sight channel in which the SMCs have large angular spreads and no SMCs are exceedingly powerful compared to the others. This kind of multipath profile promotes decorrelation between the signals received on the different $\mathrm{Rx}$ array elements. In the high SNR regime, the constant capacity loss between the simulated channel and the channel with two independent spatial streams amounts to 15.5 Mbps.

\section{CONCLUSION AND FUTURE WORK}

This work presented a methodology to simulate the MIMO capacities of IEEE 802.11n links based on empirical multipath radio channels in a residential house. These radio channels include both specular and dense multipath components. Future work in this area of research includes:

- to perform an analysis of the MIMO capacity carried by the specular and the dense multipath components separately,

- and to correlate the simulated capacities with throughput measurements of an actual IEEE 802.11n access pointclient system in the residential house.

\section{ACKNOWLEDGMENT}

The authors would like to thank Matthias Van den Bossche, M. Eng., for his help in building the channel sounding measurement system, and Jan De Beule, M. Sc. Eng., for his help during the measurements. 
FORWARD is a project co-funded by iMinds, a digital research institute founded by the Flemish Government. Project partners are ArcelorMittal, Egemin, Excentis, Siemens, and Volvo, with project support from IWT. Emmeric Tanghe is a Post-Doctoral Fellow of the FWO-V (Research Foundation Flanders, Belgium).

\section{REFERENCES}

[1] A. Richter, "Estimation of Radio Channel Parameters: Models and Algorithms," Ph.D. dissertation, Technische Universität Ilmenau, Fakültat für Elektrotechnik und Informationstechnik, Ilmenau, DE, 2005.

[2] A. Richter, J. Salmi, and V. Koivunen, "Distributed Scattering in Radio Channels and its Contribution to MIMO Channel Capacity," in European Conference on Antennas and Propagation, Nice, FR, 2006, pp. 1-7.

[3] M. Landmann, M. Kaeske, R. Thoma, J.-I. Takada, and I. Ida, "Measurement Based Parametric Channel Modeling Considering Diffuse Scattering and Specular Components," in International Symposium on Antennas and Propagation, no. 1, 2007, pp. 153-156.

[4] M. Landmann, "Limitations of Experimental Channel Characterisation," Ph.D. dissertation, Technische Universität Ilmenau, Fakültat für Elektrotechnik und Informationstechnik, Ilmenau, DE, 2008.

[5] E. Tanghe, D. P. Gaillot, M. Liénard, L. Martens, and W. Joseph, "Experimental Analysis of Dense Multipath Components in an Industrial Environment," IEEE Transactions on Antennas and Propagation, vol. 62, no. 7, pp. 3797-3805, Jul. 2014. 